\title{
Phase-resolved imaging of confined exciton-polariton wave functions in elliptical traps
}

\author{
Gaël Nardin, Yoan Léger, Barbara Pietka, François Morier-Genoud, and Benoît Deveaud-Plédran \\ Laboratoire d'Optoélectronique Quantique, École Polytechnique Fédérale de Lausanne (EPFL), Station 3, \\ CH-1015 Lausanne, Switzerland
}

(Received 4 March 2010; revised manuscript received 11 June 2010; published 7 July 2010)

\begin{abstract}
We study the wave functions of exciton polaritons trapped in the elliptical traps of a patterned microcavity. A homodyne detection setup with numerical off-axis filtering allows us to retrieve the amplitude and the phase of the wave functions. Doublet states are observed as the result of the ellipticity of the confinement potential and are successfully compared to even and odd solutions of Mathieu equations. We also show how superpositions of odd and even states can be used to produce "donut" and "eight-shape" states which can be interpreted as polariton vortices.
\end{abstract}

DOI: 10.1103/PhysRevB.82.045304

PACS number(s): 71.36.+c, 42.55.Sa, 71.35.-y, 42.30.Rx

\section{INTRODUCTION}

Exciton polaritons are hybrid light-matter quasiparticules arising from the strong coupling between the electromagnetic mode of a semiconductor microcavity and quantum well excitons. ${ }^{1}$ While their matter part allows for a wide range of nonlinear behaviors, ${ }^{2-4}$ their photonic component provides the polaritons with a very small effective mass and allows for straightforward optical excitation and detection of the polariton field. ${ }^{5}$ A direct consequence of their small effective mass (or large de Broglie wavelength) is the possibility to confine polaritons in traps of sizes in the micrometer range. ${ }^{6-8} \mathrm{Im}$ ages of the two-dimensional distribution of the confined states probability density can be realized either using a tomography technique ${ }^{9,10}$ or by directly imaging the coherent emission of a given state when it is resonantly excited with a continuous wave laser. ${ }^{10,11}$ The emission intensity gives direct information on the probability density of the confined polariton states. In this contribution we are demonstrating a method for imaging the full wave function, i.e., its amplitude and phase, using a homodyne detection scheme. We use it to image the wave functions of polaritons confined in the elliptical traps of a patterned semiconductor microcavity. Elliptical geometries are a topic of interest in optics when one wants to characterize the transverse patterns of elliptical laser beams. In this scope, Gutiérrez-Vega et al. have predicted and demonstrated Mathieu and Ince-Gaussian beams. ${ }^{12-15}$ However the measurement of the phase structure still remains a missing component. We show here that the measured amplitude and phase structures of our confined microcavity polaritons can be successfully compared to even and odd solutions of Mathieu equations. We also evidence combinations of even and odd states which result in "donut" or "eight-shape" states featuring the topology of polariton vortices. This phase-resolved measurement technique might also be applied to probe the eigenmodes of empty optical resonators. Indeed, although all our measurements are performed in the strong-coupling regime, similar patterns would be observed for the scattering of light on empty elliptical micropillars.

\section{SAMPLE DESCRIPTION}

The sample under study is a patterned GaAs $\lambda$ cavity with one embedded InGaAs quantum well (excitonic resonance at
$1.484 \mathrm{eV}$ ), sandwiched between two semiconductor distributed Bragg reflectors (DBRs), featuring a vacuum Rabi splitting of $3.5 \mathrm{meV}$. Polariton traps consist of elliptical mesas that were etched on the microcavity spacer [see Fig. 1(a)], in order to create a lateral confining potential for the cavity electromagnetic field. ${ }^{16}$ The electromagnetic field is therefore confined in the three dimensions: in the growth direction due to the DBRs and in the in-plane directions due to the local confinement potential created by the mesa. Strong coupling between the confined cavity modes and the excitonic resonance produces confinement for both upper and lower polariton branches, leading to discrete confined polariton states. ${ }^{8,9}$ Figure 1(b) shows a spatially resolved photoluminescence spectrum of the polariton states confined in a mesa of $10 \mu \mathrm{m}$ mean diameter, under nonresonant pumping. Discrete confined states can be observed for upper and lower polariton branches. The linewidth of these states is on the order of $80 \mu \mathrm{eV}$. All measurements presented in the paper were performed on lower polariton states confined in elliptical mesas of $10 \mu \mathrm{m}$ mean diameter, and for a detuning of $\delta \sim 0$ meV between the confined photonic mode and the excitonic resonance. Similar results were obtained in traps with mean diameters varying between 3 and $20 \mu \mathrm{m}$, as well as on the confined upper polariton branch. All results have been obtained under low power resonant optical pumping, in the linear regime. Nonlinear emission and relaxation of the confined states were, however, demonstrated in previous publications on the same sample, ${ }^{17-19}$ but are not exploited in the present contribution.

\section{SETUP}

In order to image the wave function of the confined polariton states rather than their probability density only, we need a phase-resolved detection scheme. In this perspective, we built a homodyne detection setup [Fig. 1(c)]. We split the beam of a continuous wave (cw) Ti-Sapphire laser into two parts: one part was used to resonantly excite the polariton states from the back of the sample using a camera objective, the other part served as a phase reference. The sample was held in a cryostat at a temperature of $\sim 4 \mathrm{~K}$ and the Gaussian excitation spot had a diameter of $15 \mu \mathrm{m}$. Scanning the excitation energy allows to selectively excite the eigenstates of 


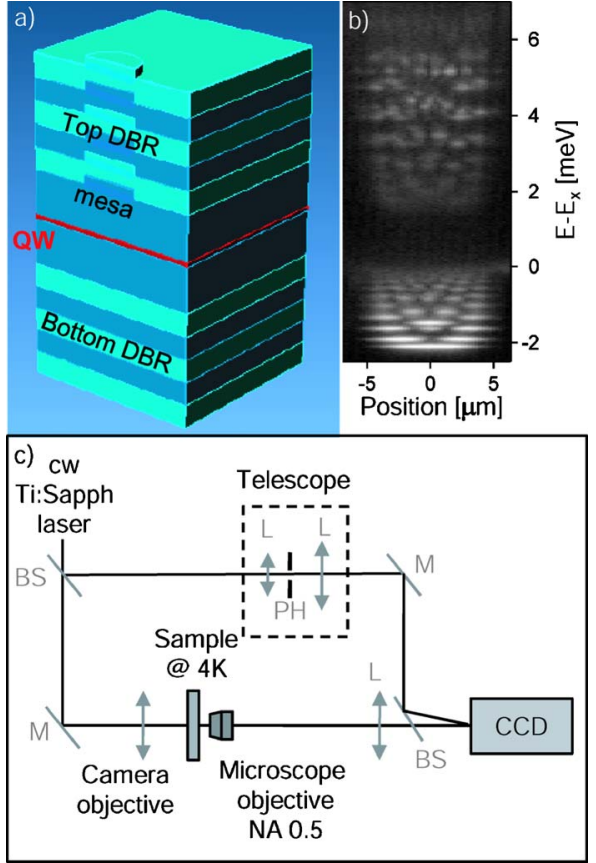

FIG. 1. (Color online) (a) Scheme of the microcavity sample with mesa (not to scale, only a few DBR pairs are shown). (b) Spatially resolved photoluminescence spectrum of the polaritons confined in a mesa of $10 \mu \mathrm{m}$ mean diameter, under nonresonant pumping. Energy is plotted with respect to the bare exciton energy $\left(E_{x}=1.484 \mathrm{eV}\right)$. Discrete confined states are visible for lower (below $E_{x}$ ) and upper (above $E-E_{x}=1.5 \mathrm{meV}$ ) polariton branches. (c) Scheme of the homodyne detection setup. BS stands for beam splitter, $\mathrm{M}$ for mirror, $\mathrm{L}$ for lens, and $\mathrm{PH}$ for pinhole.

the trap. When an eigenstate was found, fine tuning of the excitation position and incidence angle was made in order to maximize the coupling between the pump and the eigenstate, by maximizing the coherent emission intensity. We made sure that this fine tuning did not affect the shape of the state. The light transmitted by the polariton mode was then collected using a 0.5 NA microscope objective and imaged on a CCD. Depending on the position of the imaging lens, real or reciprocal space images could be obtained. The reference arm was sent to a telescope for beam enlargement and wavefront tuning, and interfered with the signal on the CCD. A slightly different incidence angle was used for the signal and the reference, in order to provide straight interference fringes. After a numerical Fourier transform of the interferogram, we were able to differentiate the interference term from the continuous part (see Appendix A for more details). Using off-axis filtering, ${ }^{20}$ we could then keep the interference term only, and extract the amplitude and phase of the polariton states.

This phase and amplitude extraction procedure is demonstrated in Figs. 2(a)-2(c), for the ground state $(n=1, m=0)$ of the trapped lower polariton. Figure 2(a) presents the result of the interference of the real-space coherent emission with the reference beam. The extracted amplitude and phase of the wave function are presented in Figs. 2(b) and 2(c), respectively. The phase gradient induced by the incidence angle difference between the signal and reference arms can be de-

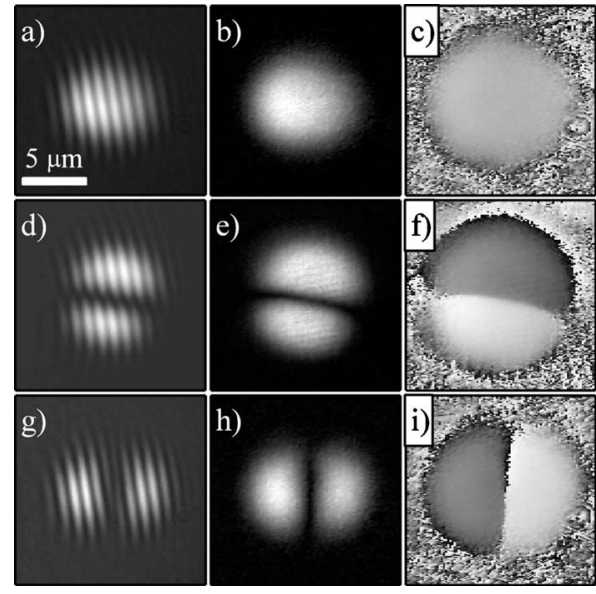

FIG. 2. (a) Real-space coherent emission of the (a) $(n=1, m$ $=0$ ) lower polariton state in a $10 \mu \mathrm{m}$ mean diameter mesa, interfered with the reference beam. (b) Amplitude and (c) phase of the state wave function, extracted using off-axis filtering. (d)-(i) same as (a)-(c), but for the $(n=1, m=1)$ doublet state. For this $(n=1, m$ $=1$ ) doublet state, the phase-gradient reference used is the one that provides a constant phase for the $(n=1, m=0)$ state, as it is theoretically expected to be constant. In (f) and (i), a clear $\pi$-phase shift is visible between the two lobes, indicating that the two lobes of the wave function are of opposite sign. The combination of the amplitude and phase information provides a full information on the confined polariton wave function. Interferograms and amplitude patterns are in a linear gray scale from minimal (black) to maximal (white) intensity. Phase patterns are in a linear gray scale from 0 (black) to $2 \pi$ (white).

duced from this measurement, as the phase is supposed to be constant for this ground state. This phase gradient was then used as a reference for all the further measurements, as they share the same experimental setup alignment.

\section{DESCRIPTION OF THE CONFINED STATES AND RESULTS}

The confined states can be labeled in a circular basis by two quantum numbers $(n, m)$ with $n=1,2,3, \ldots$ and $m$ $=0,1,2, \ldots$ In polar coordinates $(r, \phi), n$ gives the number of lobes of the wave function in the radial direction. For a perfect cylindrical symmetry, there are, for a given quantum number $m$, two degenerate $\pm m$ states which undergo a phase rotation of $e^{ \pm i m \phi}$ in the azimuthal direction. However, due to the ellipticity of the trap (see inset of Fig. 3 for a picture of the mask used for the photolithography of the mesas), these $+m$ and $-m$ states will no longer be the eigenstates of the trap. Let us consider the expression of the confinement potential as $V(r, \phi)=V_{0}+\delta V(r, \phi)$. In the circular basis, the angular perturbation $\delta V(\phi)$ will provide coupling terms between states $\psi_{m}$ and $\psi_{m^{\prime}}$. The characteristic coupling energy is given by the perturbation matrix elements $W_{m, m^{\prime}}$, whose azimuthal component are given by

$$
W_{m, m^{\prime}}(\phi)=\left\langle\psi_{m}|\delta V(\phi)| \psi_{m^{\prime}}\right\rangle=\int \delta V(\phi) e^{i\left(m^{\prime}-m\right) \phi} d \phi
$$

which is nothing but the Fourier transform of the angular variation in the potential with respect to the transform vari- 


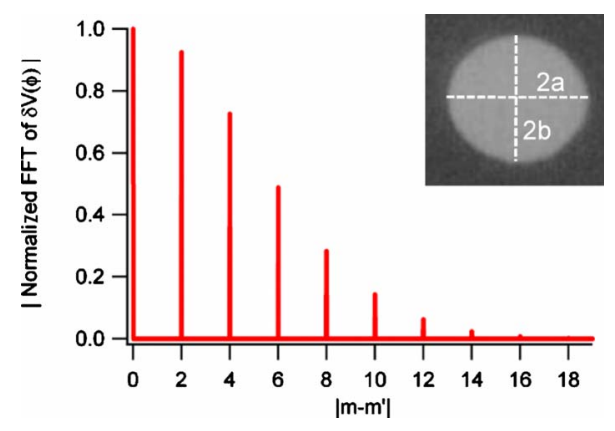

FIG. 3. (Color online) Absolute value of the fast Fourier transform (FFT) of the angular expression of the elliptical confinement potential $\delta V^{n}(\phi)$ [defined in Eq. (2) with $n=100$, and using $a$ and $b$ as measured in inset]. The FFT is plotted with respect to the parameter $\left|m-m^{\prime}\right|$ in order to express the coupling between states $\psi_{m}$ and $\psi_{m^{\prime}}$. We can see that the coupling is nonzero only for states satisfying $\left|m-m^{\prime}\right|=2 u$, where $u \in \mathbb{N}$, and that it is monotonically decreasing over this set of values. The amplitude of the FFT is normalized to its cw component. Inset: picture of the mask used for the photolithography of the polariton traps. We measured the elliptical major and minor axes $2 a$ and $2 b$ to be $2 a=10.78 \mu \mathrm{m}$ and $2 b$ $=9.27 \mu \mathrm{m}$. The corresponding eccentricity is $\epsilon=0.51$.

able $\left(m-m^{\prime}\right) / 2 \pi$. This $\delta V$ can be evaluated in the following way: let us describe, as an example, the confinement potential as an infinite elliptic potential well $V(r, \phi)$ $=\lim _{n \rightarrow \infty}\left(\frac{r}{r_{\phi}}\right)^{n} V^{*}$, where $r_{\phi}$ is the azimuthal dependence of the potential radius, given by

$$
r_{\phi}=\frac{a b}{\sqrt{(a \sin \phi)^{2}+(b \cos \phi)^{2}}}
$$

(with $a$ and $b$ semimajor and semiminor axes of the elliptical confinement measured from the inset in Fig. 3), and $V^{*}$ has the dimension of energy. It is then possible to express the perturbation of the confining potential as

$$
\begin{aligned}
\delta V(r, \phi) & =V^{*} \lim _{n \rightarrow \infty}\left[\left(\frac{r}{r_{\phi}}\right)^{n}-\left(\frac{r}{\frac{a+b}{2}}\right)^{n}\right] \\
& =V^{*} \lim _{n \rightarrow \infty}\left[r^{n}[\underbrace{\left.r_{\phi}^{-n}-\left(\frac{a+b}{2}\right)^{-n}\right\}}_{\equiv \delta V^{n}(\phi)}] .\right.
\end{aligned}
$$

The Fourier transform of the angular variation in the confinement potential $\delta V^{n}(\phi)$ is presented in Fig. 3, for $n=100$. It shows that the coupling is nonzero only for states satisfying $\left|m-m^{\prime}\right|=2 u$, where $u \in \mathbb{N}$, and that it is monotonically decreasing over this set of values. A detailed analysis would reveal that for very small deviation from the cylindrical symmetry, this decrease is exponential, and that therefore the only important coupling occurs between states with quantum numbers satisfying $\left|m-m^{\prime}\right|=2$. For this reason, in previous publications, ${ }^{9,11}$ we considered, in a first approximation, that the only significant coupling was between $m=+1$ and $m=-1$ states (the coupling between $m=0$ and $m=2$ states will be discussed later), and experimentally observed that the perturbation of the cylindrical symmetry results in a degeneracy lift of the $m= \pm 1$ doublet into two new states $\psi_{ \pm}$ $=\frac{1}{\sqrt{2}}\left[e^{+i \phi} \pm e^{-i \phi}\right]$, featuring $|2 m|=2$ lobes in the azimuthal direction, aligned along the ellipse axes.

This $(n=1, m=1)$ doublet can be observed in Figs. 2(d) and $2(\mathrm{~g})$ display the interferograms obtained for the $\psi_{ \pm}$ states with the corresponding amplitudes [Figs. 2(e) and 2(h)] and phases [Figs. 2(f) and 2(i)].

We measured the energy separation between these two states, using spatially resolved spectroscopy as described in Refs. 9 and 10, to be on the order of $50 \mu \mathrm{eV}$ (with a resolution limit of $30 \mu \mathrm{eV}$ ). On the phase structure, a clear $\pi$-phase shift between the two lobes of the wave function, indicating that the two lobes are of opposite sign. By this way we have obtained the full information about the wave function of the confined state.

Figure 3 shows that for the values $a$ and $b$ measured in the inset, we are, in fact, in the case of a pronounced ellipticity, inducing non-negligible coupling terms for $\left|m-m^{\prime}\right|$ $=0,2,4,6, \ldots$ It indicates that, even if it is less pronounced than for the \pm 1 states, there should be a finite coupling between $m=+2$ and $m=-2$ states. This is what we actually observe on the states characterized by the quantum numbers $(n=1, m= \pm 2)$. We can see in Figs. 4(a)-4(d) that there is a doublet structure separated by a splitting of the order of or smaller than our resolution limit of $30 \mu \mathrm{eV} .^{21}$ Moreover, a significant qualitative deviation from the patterns expected in a circular geometry is observed on the amplitude patterns [Figs. 4(a) and 4(c)] and phase structures [Figs. 4(b) and 4(d)].

Indeed, the state shown in Figs. 4(c) and 4(d) does not feature a plain four-lobe pattern. In order to understand this feature, we need to solve the wave equation on the actual elliptic domain. The two-dimensional wave equation is a Helmholtz equation

$$
\nabla^{2} \psi+k^{2} \psi=0
$$

whose eigenvalues $k$ are related to the energy of the eigenmodes $\psi$. In terms of the elliptical coordinates $(\xi, \eta)$, variables can be separated and one obtains two equations known as the angular and radial Mathieu equations ${ }^{22}$ (see Appendix B for more details on Mathieu equations and their resolution). Each of them has two families of independent solutions, namely, even and odd Mathieu functions. The complete two-dimensional even (odd) solutions, analogous to the standing waves of a vibrating membrane, are then given by the product of the angular even (odd) Mathieu function by the radial even (odd) Mathieu function. ${ }^{23-25}$ These eigenstates are also characterized by two quantum numbers, which also provide the number of zeros in radial and azimuthal directions, respectively. For these reasons we will still use the quantum numbers $n$ and $m$ and indicate the parity of the state [e.g., $(n=1, m=2, e)$ for the $(n=1, m=2)$ even state] to describe the eigenstates. These eigenstates should not be confused with the $(n, \pm m)$ states, which were eigenstates of the circular trap. The even and the odd two-dimensional solutions of Mathieu equations for the quantum numbers ( $n$ $=1, m=2$ ) have been computed and are presented in Figs. 


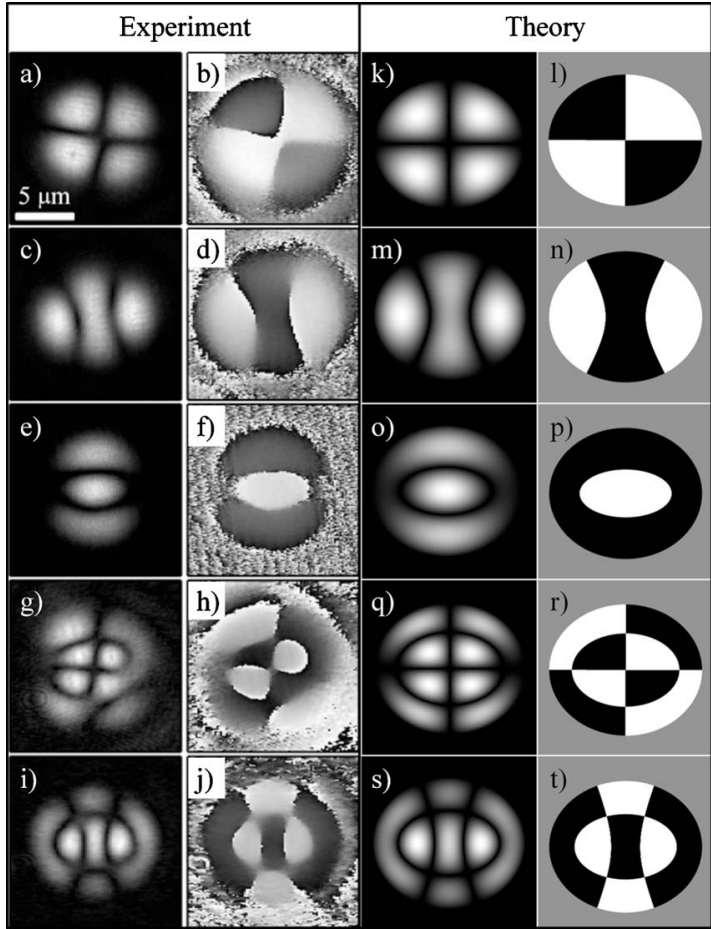

FIG. 4. Real-space experimental images (obtained by the same method than for Fig. 2) of the amplitude and phase of the [(a) and (b) $[(n=1, m=2, o)$ state, [(c) and (d)] $(n=1, m=2, e)$ state, [(e) and (f)] $(n=2, m=0)$ state, $[(\mathrm{g})$ and (h)] $(n=2, m=2, o)$ state, and [(i) and $(\mathrm{j})](n=2, m=2, e)$ state (Ref. 26). The phase-gradient reference used is the one that provides a constant phase for the $(n=1, m=0)$ state of the trap. Amplitude patterns are in a linear gray scale from minimal (black) to maximal (white) intensity. Phase patterns are in a linear gray scale from 0 (black) to $2 \pi$ (white). Last two columns: corresponding amplitude and phase structures obtained from the analytical solutions of the wave equation. The boundary conditions are given by the Dirichlet condition (see Appendix B) on the elliptical domain defined by the values measured in the inset of Fig. 3(b). The amplitude structures are given by the absolute value of the wave function. For the phase structures, black is plotted for the negative parts of the wave function and white for the positive parts.

4(k) and 4(l) (odd) and $(m)-(n)$ (even). We have used the parameters measured in the inset of Fig. 3 for defining the confinement geometry. There is an excellent qualitative agreement between these analytical solutions and the experimentally observed patterns for both the phase and the amplitude. It can be observed that the nodal lines of the wave functions follow confocal ellipses ( $\xi=$ constant) and hyperbolas $(\eta=$ constant $)$.

We mentioned above that according to our description of the coupling between states using the Fourier transform of the elliptical confinement, we expect a coupling between $m$ $=0$ and $m= \pm 2$ states. It means that $m=0$ states patterns should also be affected by the ellipticity of the trap. This qualitative alteration can be observed on the amplitude and phase structures of the $(n=2, m=0)$ state presented in Figs. 4(e) and 4(f). The perturbation can be again well reproduced with the analytical solutions of Mathieu equations [Figs. 4(o) and $4(\mathrm{p})]$.
Eventually, the experimental and theoretical patterns for the $(n=2, m=2)$ doublet are presented in Figs. 4(g)-4(j) and $4(\mathrm{q})-4(\mathrm{t})$, showing again an excellent agreement between experiment and theory.

Solving the wave equation on the elliptical domain also allows to obtain a theoretical energy spectrum. The most straightforward physical approach is to describe the system with the time-independent Schrödinger equation. ${ }^{9}$ The eigenvalues $k$ of Eq. (3) are related to the physical parameters by $k^{2}=\frac{2 m^{*} E}{\hbar^{2}}$, where $m^{*}$ is the effective mass of the polariton and $E$ is the eigenenergy with respect to the bottom of the confinement potential. However, using these physical parameters, the experimental spectrum cannot be satisfactorily reproduced. This is probably due to the two following reasons: first, with the Dirichlet condition, we have assumed an infinite potential barrier created by the trap whereas the effective confinement potential for the lower polariton is finite, on the order of $3 \mathrm{meV}$; second, the effective mass of the polariton depends of the detuning of the given polariton mode with respect to the excitonic resonance and is therefore different for each confined polariton mode. Another more complicated approach, consisting in first solving the Maxwell equation to find the confined optical modes and then strongly coupling these modes to the exciton, ${ }^{8,27}$ would allow to overcome the issue of the variable effective mass, but not of the finite potential boundary, which would require to use the evanescent radial Mathieu functions (RMF) of the second kind $K e_{m}$ and $K o_{m}$. Nevertheless, without going further into the physical modeling of the system in order to obtain absolute values for the eigenenergies, we can learn qualitative information about the splitting between even and odd states with respect to the quantum numbers $(n, m)$. The computation of the RMF (see Fig. 7 and Appendix B) indicates that for a given $n$, the energy splitting between even and odd states is higher for $m=1$ than for $m=2$. Using the values for the elliptical axes $a$ and $b$ measured from the inset of Fig. 3, we find through the computation of the RMF a splitting of $48 \mu \mathrm{eV}$ for the $(n$ $=1, m=1)$ doublet and of $31 \mu \mathrm{eV}$ for the $(n=1, m=2)$ doublet. These values are in very good agreement with the measured values $(\sim 50 \mu \mathrm{eV}$ and $\sim 30 \mu \mathrm{eV}$, respectively). Another qualitative information (also visible in Fig. 7) is that for a given order $m$, the energy splitting between even and odd states is increasing with $n$.

We would like to mention another category of wave functions that we can excite in the mesas. The energy splitting between even and odd wave functions being on the order of the state linewidths, it is possible, by pumping at an intermediate energy, to excite a combination of the two split states, and therefore overcome the $\pm m$ splitting (the resulting combination is therefore not an eigenstate of the elliptic trap). The kind of superposition will depend on the excitation conditions. An excitation focused on the mesa will excite both $+m$ and $-m$ states, from which the interference pattern will be locked by the position of the laser. This has been used to control the resulting wave-function pattern that features $|2 m|$ lobes. ${ }^{11}$ On the other hand, an excitation beam with finite incidence angle, focused on the side of the mesa, can be used to create pure $+m$ or $-m$ states. ${ }^{28}$ The emission patterns obtained using these specific pumping conditions are presented in Figs. 5(a)-5(c) for a superposition of $(n=1, m=1, e)$ and 


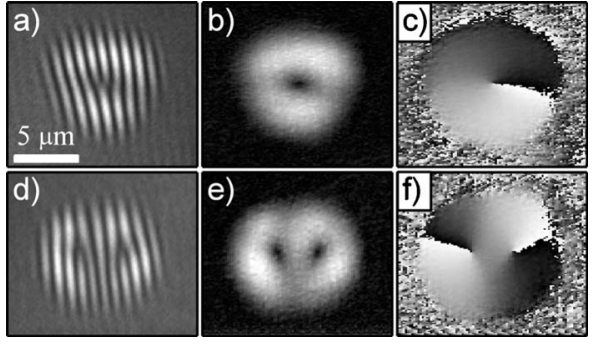

FIG. 5. (a) Interferogram obtained from the interference of the coherent emission of a $(n=1, m=1)$ donut state with the reference beam. A clear forklike dislocation is visible, sign of a phase singularity. [(b) and (c)] Extracted amplitude and phase structures, showing a vortexlike structure. (d)-(f) same as (a)-(c) but for the $(n$ $=1, m=2)$ state. The two phase singularities of this eight shape are located at the intersections of the nodal lines of $(n=1, m=2, e)$ and $(n=1, m=2, o)$ states. Interferograms and amplitude patterns are in a linear gray scale from minimal (black) to maximal (white) intensity. Phase patterns are in a linear gray scale from 0 (black) to $2 \pi$ (white).

$(n=1, m=1, o)$. The interferogram presented in Fig. 5(a) is characterized by a clear forklike dislocation in its center, sign of a phase singularity. The extracted amplitude and phase patterns show then a minimum of the polariton density at the position of the singularity (core), and a $2 \pi$-phase shift around the singularity. This superposition carries an integer angular orbital momentum $m=+1$. Its topological structure is identical to a vortex in the polariton gas, although it is created by geometrical means and is not linked with the concept of superfluidity. By analogy to laser transverse mode patterns, this polariton vortex can also be seen as a $T E M_{01^{*}}$ donut mode, resulting of a superposition of $T E M_{10}$ and $T E M_{01}$ modes, as shown in Refs. 29-31. Figures $5(\mathrm{~d})-5(\mathrm{f})$ show the superposition of $(n=1, m=2, e)$ and $(n=1, m=2, o)$. The expected double topological charge is split into two single vortices due to the ellipticity of the trap. The positions of the two cores are given by the intersections of the nodal lines of $(n=1, m=2, e)$ and $(n=1, m=2, o)$ state patterns.

It is also possible to image the polariton emission in the Fourier plane, simply by changing the position of the imaging lens. Interference of reciprocal space images with the reference arm provides the same type of interferograms than for real-space imaging from which we can extract amplitude and phase of the wave functions as well. A series of reciprocal space patterns for four different states is presented in Fig. 6. We can see that patterns in the Fourier plane are very similar than real-space patterns, except for the fact that higher order diffraction is visible around the zero-order pattern. The homodyne detection allows to observe the expected $\pi$-phase shift between two consecutive diffraction orders.

\section{CONCLUSION}

To summarize, we developed a homodyne detection setup that allows for the imaging in real and reciprocal space of the complete wave-function patterns (amplitude and phase) of microcavity polaritons. We have used this setup to record

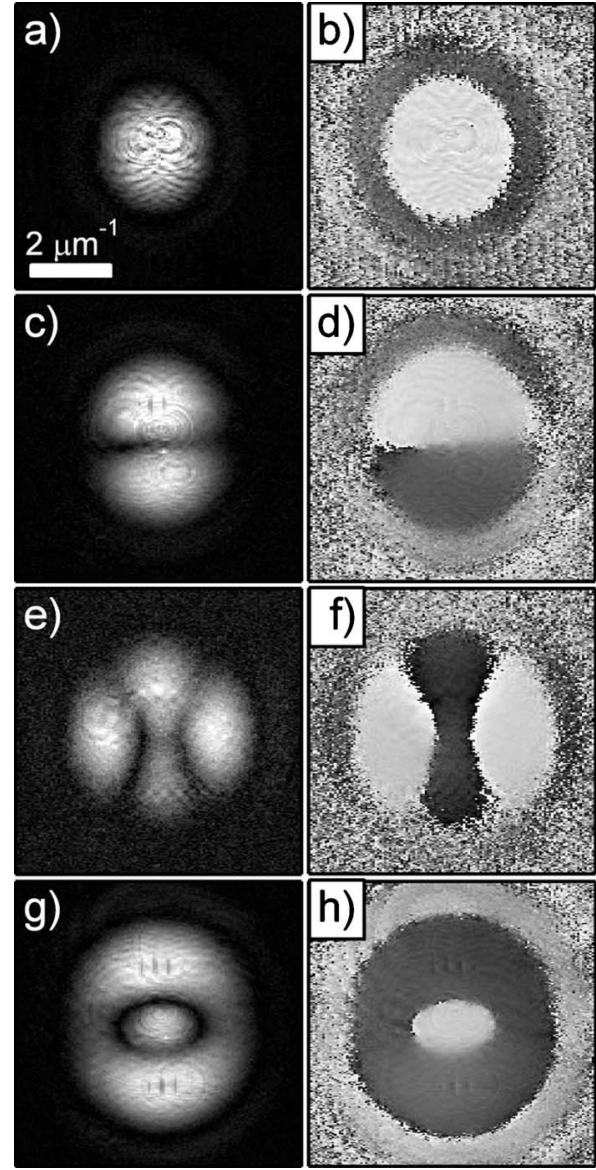

FIG. 6. Experimental amplitude and phase patterns in the Fourier plane, for [(a) and (b)] $(n=1, m=0)$ state, $[(\mathrm{c})$ and (d)] ( $=1, m=1, e)$ state, $[(\mathrm{e})$ and (f) $(n=1, m=2, e)$ state, and $[(\mathrm{g})$ and (h)] $(n=2, m=0)$ state. Higher diffraction orders can be seen around the main patterns. Amplitude patterns are in a linear gray scale from minimal (black) to maximal (white) intensity. Phase patterns are in a linear gray scale from 0 (black) to $2 \pi$ (white).

images of polariton states confined in the elliptical traps of a patterned microcavity. We could observe the significant effect of the ellipticity of the confining potential on the wavefunction patterns, which were successfully compared to analytical solutions described in terms of even and odd solutions of Mathieu equations. We have also produced superpositions of even and odd states which take the form of donut and eight-shape wave functions and are topologically identical to polariton vortices. This shows the possibility of creating vortices in a coherently pumped polariton gas by geometrical means only, in absence of superfluidity.

\section{ACKNOWLEDGMENTS}

We would like to thank R. Cerna, T. K. Paraïso, K. G. Lagoudakis, O. El Daif, N. Pavillon, and B. Caire-Remonnay for helpful discussions. We acknowledge support by the Swiss National Research Foundation through the "NCCR Quantum Photonics." 


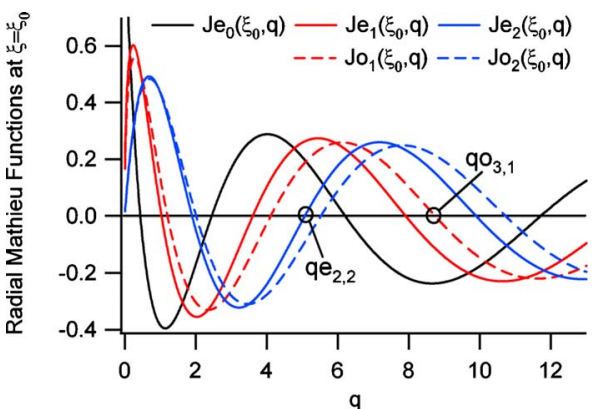

FIG. 7. (Color online) Plot of even and odd RMF $J e_{m}\left(\xi_{0}, q\right)$ and $J o_{m}\left(\xi_{0}, q\right)$, as a function of the parameter $q$, at the boundary of the potential trap $\left(\xi_{0}=1.2931\right)$, for $m=0,1,2$. The parameter $q$ of the eigenmodes satisfying the Dirichlet condition are given by the zeros of the RMF. $q e_{n, m}$ and $q o_{n, m}$ are the $n$th zero of even and odd RMF of order $m$. The energies or frequencies of the eigenmodes are proportional to $q$. We can see that for a given $n$, the energy splitting between even and odd states is higher for $m=1$ than for $m=2$. For a given order $m$, the energy splitting is increasing with $n$.

\section{APPENDIX A: DETAILS OF THE PHASE EXTRACTION PROCEDURE}

We detail here the phase-extraction procedure. When an interferogram is recorded by the CCD camera, the detected intensity is proportional to $\left\langle\left(E_{s}+E_{r}\right)^{2}\right\rangle=\left\langle E_{s}^{2}\right\rangle+\left\langle E_{r}^{2}\right\rangle$ $+2 E_{s_{0}} E_{r_{0}} \cos \left(\phi_{s}-\phi_{r}+\Delta \theta\right)$, where $E_{s} \equiv E_{s_{0}} e^{i \phi_{s}}$ is the signal field (with its amplitude $E_{s_{0}}$ and phase $\phi_{s}$ ), $\left\langle E_{s}^{2}\right\rangle$ the signal field intensity $\left(\langle\cdots\rangle\right.$ denotes the temporal average), $E_{r}$ $\equiv E_{r_{0}} e^{i \phi_{r}}$ the reference field, and $\Delta \theta$ the phase component induced by the difference of incidence angle between the signal and reference. The phase of the signal is not accessible from its intensity but can be extracted from the interference term $2 E_{s_{0}} E_{r_{0}} \cos \left(\phi_{s}-\phi_{r}+\Delta \theta\right)$. To isolate this contribution, we perform a numerical Fourier transform of the interferogram. In the Fourier plane, the interference term is an offaxis contribution, while the continuous terms are situated at the origin and can be filtered out. By this way, we filter out as well all incoherent contributions. We keep then only the interference term, make the realistic assumption that $E_{r}$ is constant over the measurement area, and perform an inverse Fourier transform, from which we obtain $E_{s}(x, y)$, including its amplitude and phase informations. The last step is to get rid of the phase component $\Delta \theta$ induced by the setup alignment. The procedure that we follow to remove this phase component is explained in the main text.

\section{APPENDIX B: MATHIEU EQUATIONS AND FUNCTIONS}

In this appendix we show the tools that we used to plot the eigenmodes of the polaritons confined in elliptical traps. A detailed description of the method can also be found in Ref. 24 and a comprehensive overview of Mathieu functions is available in Ref. 25.

In order to find the eigenmodes of polaritons confined in an elliptical trap, we need to solve Helmholtz Eq. (3) in elliptical coordinates $(\xi, \eta)$, which are linked to cartesian coordinates by

$$
\begin{gathered}
x=\frac{c}{2} \cosh \xi \cos \eta . \\
y=\frac{c}{2} \sinh \xi \sin \eta,
\end{gathered}
$$

where $c$ is the distance between the two foci of the ellipse and is given by $\frac{c}{2}=\sqrt{a^{2}-b^{2}}$ with $a$ and $b$ semimajor and semiminor axes of the elliptical confinement, respectively. The eccentricity $\epsilon$ of elliptical domain is given by $\epsilon=\frac{c}{2 a}$. The domain boundary is defined by $\xi=\xi_{0}$, such as $a=\frac{c}{2} \cosh \xi_{0}$ or $b=\frac{c}{2} \sinh \xi_{0}$. Ensembles of points with $\xi=$ const represent confocal ellipses and ensembles of points with $\eta=$ const represent confocal hyperbolas. After performing the separation of variables $\psi=R(\xi) \Phi(\eta)$, one obtains two equations

$$
\begin{aligned}
& \frac{d^{2} \Phi}{d \eta^{2}}+(a-2 q \cos 2 \eta) \Phi=0 \\
& \frac{d^{2} R}{d \xi^{2}}-(a-2 q \cos 2 \xi) \Phi=0,
\end{aligned}
$$

where $q=\frac{1}{16} c^{2} k^{2}$ and $a$ is a separation variable. Equation (B3) is known as the ordinary or angular Mathieu equation and Eq. (B4) as the modified or radial Mathieu equation. Solutions of the angular Mathieu Eq. (B3) form two independent families of solutions, even and odd angular Mathieu functions $(\mathrm{AMF})$

$$
\Phi_{m}=\left\{\begin{array}{lll}
c e_{m}(\eta, q) & m=0,1,2, \ldots & (\text { even AMF }) \\
s e_{m}(\eta, q) & m=1,2,3, \ldots & (\text { odd AMF }),
\end{array}\right.
$$

where $m$ is the order of the function. The AMF satisfy the periodic boundary conditions on $\eta \in[0,2 \pi]$, and feature $2 m$ zeros on this interval. There is therefore a clear analogy between the order $m$ and the quantum number $m$ defined in Sec. IV. Solutions of the radial Mathieu Eq. (B4) which satisfy the continuity conditions at $\xi=0$ are even and odd RMF of the first kind

$$
R_{m}=\left\{\begin{array}{lll}
J e_{m}(\eta, q) & m=0,1,2, \ldots & (\text { even } \mathrm{RMF}) \\
J o_{m}(\eta, q) & m=1,2,3, \ldots & \text { (odd RMF) }
\end{array}\right.
$$

In order to find the eigenmodes of Helmholtz Eq. (3) we need to find the values of $q$ that satisfy the Dirichlet condition

$$
J e_{m}\left(\xi_{0}, q\right)=0 \text { or } J o_{m}\left(\xi_{0}, q\right)=0 .
$$

There is an infinite family of solutions $q_{n, m}$ that satisfy this condition. We denote $q e_{n, m}$ and $q o_{n, m}$ the $n$th zero of $J e_{m}$ and $J o_{m}$, respectively. There is an analogy between this number $n$ and the quantum number $n$ defined in Sec. IV. Figure 7 displays $J e_{m}\left(\xi_{0}, q\right)$ and $J o_{m}\left(\xi_{0}, q\right)$ (Ref. 32) for $m=0,1,2$, where the elliptical boundary $\xi_{0}=1.2931$ is obtained from the measurement of major axis $2 a=10.78$ and minor axis $2 b$ $=9.27$ obtained from the inset of Fig. 3, providing an eccentricity of $\epsilon=0.51$. To every $q_{n, m}$ can be associated an eigen 
value $k$, solution of Helmholtz Eq. (3), that can be linked to a frequency or energy by using the physical parameters of the problem. The even and the odd eigenmodes are given by the product of radial and angular Mathieu functions

$$
\psi e_{n, m}=J e_{m}(\xi, q) c e_{m}(\eta, q) \text { with } q=q e_{n, m},
$$

$$
\psi o_{n, m}=J o_{m}(\xi, q) \operatorname{se}_{m}(\eta, q) \text { with } q=q o_{n, m} .
$$

The amplitude and sign (black for negative, white for positive) of the eigenmodes are presented in third and fourth columns of Fig. 4, respectively, for comparison with experimental measurements. An excellent qualitative agreement is observed.
${ }^{1}$ C. Weisbuch, M. Nishioka, A. Ishikawa, and Y. Arakawa, Phys. Rev. Lett. 69, 3314 (1992).

${ }^{2}$ C. Ciuti, P. Schwendimann, B. Deveaud, and A. Quattropani, Phys. Rev. B 62, R4825 (2000).

${ }^{3}$ M. Saba, C. Ciuti, J. Bloch, V. Thierry-Mieg, R. André, L. S. Dang, S. Kundermann, A. Mura, G. Bongiovanni, J. L. Staehli, and B. Deveaud, Nature (London) 414, 731 (2001).

${ }^{4}$ A. Baas, J. Ph. Karr, H. Eleuch, and E. Giacobino, Phys. Rev. A 69, 023809 (2004).

${ }^{5}$ V. Savona, C. Piermarocchi, A. Quattropani, P. Schwendimann, and F. Tassone, Phase Transitions 68, 169 (1999).

${ }^{6}$ J. Bloch, R. Planel, V. Thierry-Mieg, J.-M. Gérard, D. Barrier, J.-Y. Marzin, and E. Costard, Superlattices Microstruct. 22, 371 (1997).

${ }^{7}$ M. Obert, J. Renner, A. Forchel, G. Bacher, R. André, and L. S. Dang, Appl. Phys. Lett. 84, 1435 (2004).

${ }^{8}$ R. I. Kaitouni, O. El Daif, A. Baas, M. Richard, T. Paraiso, P. Lugan, T. Guillet, F. Morier-Genoud, J. D. Ganière, J. L. Staehli, V. Savona, and B. Deveaud, Phys. Rev. B 74, 155311 (2006).

${ }^{9}$ G. Nardin, T. K. Paraïso, R. Cerna, B. Pietka, Y. Léger, O. El Daif, F. Morier-Genoud, and B. Deveaud, Appl. Phys. Lett. 94, 181103 (2009).

${ }^{10}$ G. Nardin, R. Cerna, T. K. Paraïso, B. Pietka, Y. Léger, O. El Daif, F. Morier-Genoud, and B. Deveaud-Plédran, Superlattices Microstruct. 47, 207 (2010).

${ }^{11}$ R. Cerna, D. Sarchi, T. K. Paraïso, G. Nardin, Y. Léger, M. Richard, B. Pietka, O. El Daif, F. Morier-Genoud, V. Savona, M. T. Portella-Oberli, and B. Deveaud-Plédran, Phys. Rev. B 80, 121309 (2009).

${ }^{12}$ J. C. Gutiérrez-Vega, M. D. Iturbe-Castillo, and S. ChávezCerda, Opt. Lett. 25, 1493 (2000).

${ }^{13}$ J. C. Gutiérrez-Vega, M. D. Iturbe-Castillo, G. A. Ramírez, E. Tepichín, R. M. Rodríguez-Dagnino, S. Chávez-Cerda, and G. H. C. New, Opt. Commun. 195, 35 (2001).

${ }^{14}$ M. A. Bandres and J. C. Gutiérrez-Vega, Opt. Lett. 29, 144 (2004).

${ }^{15}$ U. T. Schwarz, M. A. Bandres, and J. C. Gutiérrez-Vega, Opt. Lett. 29, 1870 (2004).

${ }^{16}$ O. El Daïf, A. Baas, T. Guillet, J.-P. Brantut, R. Idrissi Kaitouni,
J. L. Staehli, F. Morier-Genoud, and B. Deveaud, Appl. Phys. Lett. 88, 061105 (2006).

${ }^{17}$ T. K. Paraïso, D. Sarchi, G. Nardin, R. Cerna, Y. Leger, B. Pietka, M. Richard, O. El Daïf, F. Morier-Genoud, V. Savona, and B. Deveaud-Plédran, Phys. Rev. B 79, 045319 (2009).

${ }^{18}$ O. El Daif, G. Nardin, T. K. Paraïso, A. Baas, M. Richard, J.-P. Brantut, T. Guillet, F. Morier-Genoud, and B. Deveaud-Plédran, Appl. Phys. Lett. 92, 081910 (2008).

${ }^{19}$ G. Nardin, O. El Daif, T. K. Paraïso, A. Baas, M. Richard, F. Morier-Genoud, and B. Deveaud, Phys. Status Solidi C 5, 2437 (2008).

${ }^{20}$ This technique is known as off-axis digital holography. See, for example, E. Cuche, P. Marquet, and C. Depeursinge, Appl. Opt. 38, 6994 (1999).

${ }^{21}$ For this reason, we did not notice this splitting in previous tomography experiments of Refs. 9 and 10 .

${ }^{22}$ E. Mathieu, J. Math. Pure Appl. 13, 137 (1868).

${ }^{23}$ G. Chen, P. J. Morris, and J. X. Zhou, SIAM Rev. 36, 453 (1994).

${ }^{24}$ J. C. Gutiérrez-Vega, S. Chávez-Cerda, and R. M. RodrígezDagnino, Rev. Mex. Fis. 45, 613 (1999).

${ }^{25}$ J. C. Gutiérrez-Vega, R. M. Rodrígez-Dagnino, M. A. MenesesNava, and S. Chávez-Cerda, Am. J. Phys. 71, 233 (2003).

${ }^{26}$ Images in Figs. 4(i) and 4(j) were taken during a different set of measurements, but in the same conditions than images in Figs. 4(a)-4(h)

${ }^{27}$ P. Lugan, D. Sarchi, and V. Savona, Phys. Status Solidi C 3, 2428 (2006).

${ }^{28}$ G. Nardin, K. G. Lagoudakis, B. Pietka, F. Morier-Genoud, Y. Léger, and B. Deveaud-Plédran, arXiv:1001.0846 (unpublished).

${ }^{29}$ J. E. Epler, S. Gehrsitz, K. H. Gulden, M. Moser, H. C. Sigg, and H. W. Lehmann, Appl. Phys. Lett. 69, 722 (1996).

${ }^{30}$ C. Degen, I. Fischer, and W. Elsässer, Opt. Express 5, 38 (1999).

${ }^{31}$ F. B. De Colstoun, G. Khitrova, A. V. Fedorov, T. R. Nelson, C. Lowry, T. M. Brennan, B. Gene Hammons, and P. D. Maker, Chaos, Solitons Fractals 4, 1575 (1994).

${ }^{32}$ Numerical evaluation of the Mathieu functions was obtained using the SPECFUN package, available on www.mathworks.com 